\title{
Effect of Spark Plasma Sintering Temperature on Thermoelectric Properties of Grained $\mathrm{Bi}_{1.9} \mathbf{G d}_{0.1} \mathrm{Te}_{3}$ Compound
}

\author{
M. N. Yapryntsev ${ }^{a}$, A. E. Vasil'ev ${ }^{a}$, O. N. Ivanov ${ }^{a, b, *}$, and M. V. Zhezhu ${ }^{a}$ \\ ${ }^{a}$ Belgorod National Research University, Belgorod, 308015 Russia \\ ${ }^{b}$ Shukhov Belgorod State Technological University, Belgorod, 308012 Russia \\ *e-mail: Ivanov.Oleg@bsu.edu.ru \\ Received May 15, 2019; revised June 3, 2019; accepted June 3, 2019
}

\begin{abstract}
Patterns in changes of the microstructure (grain structure) and the thermoelectric properties of the $n$-type grained $\mathrm{Bi}_{1.9} \mathrm{Gd}_{0.1} \mathrm{Te}_{3}$ compound, spark-plasma-sintered at different temperatures $\left(T_{S}=690,720\right.$, $735,750,780$, and $810 \mathrm{~K}$ ), have been studied in detail. All the samples studied were highly textured along the 001 direction parallel to the pressing direction, that resulted from preferential orientation of the grains. Orientation factor characterizing a texturing degree and estimated from XRD patterns happened to be weakly $T_{S}$-dependent. Average grain size measured along the SPS pressing direction was far less as compared to that measured in the perpendicular direction. The thermoelectric properties measured for the perpendicular direction happened to be better than the same properties, but taken for the parallel direction. Of the samples sintered at different temperatures, the highest value of the thermoelectric figure-of-merit equal to $\sim 0.73$ for the perpendicular measuring orientation was found for the sample sintered at $T_{S}=750 \mathrm{~K}$. This sample is characterizing by the maximum power factor and the low enough thermal conductivity.
\end{abstract}

Keywords: thermoelectric properties, $\mathrm{Bi}_{1.9} \mathrm{Gd}_{0.1} \mathrm{Te}_{3}$ compound, spark plasma sintering

DOI: $10.1134 /$ S1063782619130219

\section{INTRODUCTION}

It is known [1] that thermoelectric efficiency of materials is defined by the thermoelectric figure-ofmerit, $Z T$, which can be expressed as $\left(S^{2} / \rho k\right) T$ (where $T$ is the absolute temperature, $S$ is the Seebeck coefficient, $\rho$ is the specific electrical resistance, and $k$ is the total thermal conductivity with contributions from crystal lattice, carriers and bipolar conductivity). At present, bismuth telluride, $\mathrm{Bi}_{2} \mathrm{Te}_{3}$, and its $n$ - and $p$-type's alloys are the best thermoelectrics for low-temperature applications [2]. However, $Z T$ of these thermoelectrics is small enough $(\leq 1)$, that often limit their large-scale using in most thermoelectric applications. Various physical and technological approaches are developing and improving to optimally combine of $S$, $\rho$, and $k$ and, hence, to maximize $Z T$ of the $\mathrm{Bi}_{2} \mathrm{Te}_{3}-$ based compounds [3-14]. One of modern technological ways to prepare such compounds is spark plasma sintering. Homogeneous and dense thermoelectric samples with controllable elemental and phase composition can be prepared via this sintering for very short time of the SPS process. Besides, the textured grained $\mathrm{Bi}_{2} \mathrm{Te}_{3}$-based compounds can be also sintered using SPS [15-24]. At the texturing, a preferential orientation of the grains can be yielded and the thermoelectric properties measured along the texturing direction or within the plane parallel to this direction hap- pen to be remarkably different. Since for the $\mathrm{Bi}_{2} \mathrm{Te}_{3}$ single crystals $\rho$ and $k$ are highly anisotropic, $Z T$ is anisotropic, too. For grained material with completely random orientation of the grains, the thermoelectric figure-of-merit coefficient is already isotropic. The anisotropy in the transport properties of the grained $\mathrm{Bi}_{2} \mathrm{Te}_{3}$-based materials can be recovered via the texturing, at least, partially. For the textured samples, $Z T$ measured in the plane parallel to the texturing direction can approach to the highest value characteristic of the single crystal.

The purpose of this paper is to find and analyze the patterns of the spark plasma sintering temperature effect on the thermoelectric properties of the $n$-type grained $\mathrm{Bi}_{1.9} \mathrm{Gd}_{0.1} \mathrm{Te}_{3}$ compounds. There are two main reasons to choose this composition to study. Firstly, rare earth element, $\mathrm{R}(\mathrm{R}=\mathrm{Lu}, \mathrm{Ce}, \mathrm{Sm}, \mathrm{Er}, \mathrm{La}, \mathrm{Gd})$ doping can be used to remarkably enhance the thermoelectric performance of $\mathrm{Bi}_{2} \mathrm{Te}_{3}$ [25-35]. Secondly, the $\mathrm{Bi}_{1.9} \mathrm{R}_{0.1} \mathrm{Te}_{3}$ composition is optimal one to get maximum $Z T$ enhancement for many $\mathrm{R}$-doped $\mathrm{Bi}_{2} \mathrm{Te}_{3}$ compounds.

\section{EXPERIMENTAL}

Microwave-solvothermal synthesis and spark plasma sintering were applied to prepare the grained 


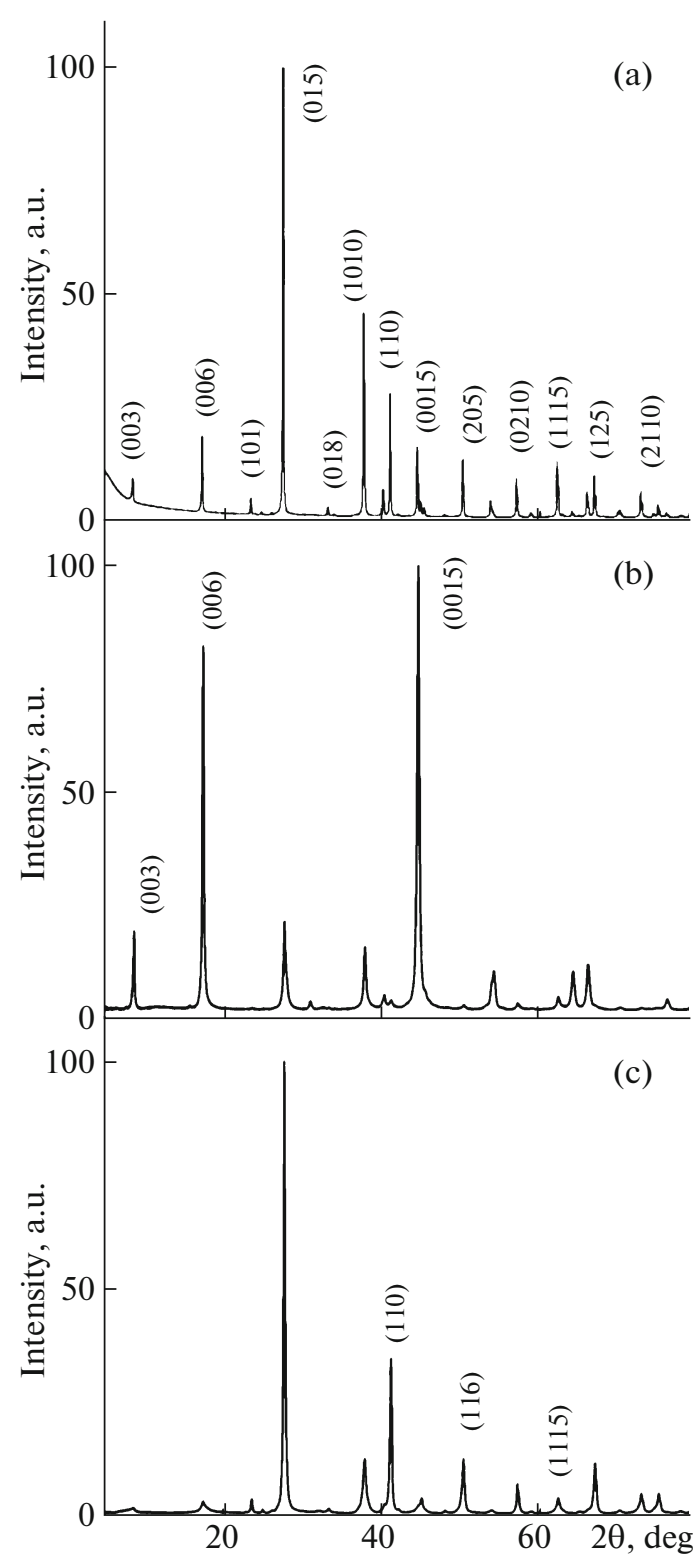

Fig. 1. (a) The XRD pattern for the starting powder. (b) The XRD pattern for the bulk sample sintered at $T_{S}=$ $750 \mathrm{~K}$ and taken on surface parallel to the pressing direction. (c) The XRD pattern for the same bulk sample taken on surface perpendicular to the pressing direction.

$\mathrm{Bi}_{1.9} \mathrm{Gd}_{0.1} \mathrm{Te}_{3}$ samples. Analytically pure chemicals $\left(\mathrm{Bi}\left(\mathrm{NO}_{3}\right)_{3} \cdot 5 \mathrm{H}_{2} \mathrm{O}, \mathrm{TeO}_{2}, \mathrm{Gd}\left(\mathrm{CH}_{3} \mathrm{COO}\right)_{3} \cdot 4 \mathrm{H}_{2} \mathrm{O}\right.$, $\mathrm{NaOH}$, poly(1-ethenylpyrrolidin-2-one), ethane1,2-diol) were used to synthesize a starting powder. First, $\mathrm{Bi}\left(\mathrm{NO}_{3}\right)_{3} \cdot 5 \mathrm{H}_{2} \mathrm{O}, \mathrm{TeO}_{2}, \mathrm{Gd}\left(\mathrm{CH}_{3} \mathrm{COO}\right)_{3} \cdot 4 \mathrm{H}_{2} \mathrm{O}$ taken in a stoichiometric ratio were dissolving in mixture of $450 \mathrm{~cm}^{3}$ ethane-1,2-diol $\mathrm{NaOH}$ and $15 \mathrm{~g}$ $\mathrm{NaOH}$ under vigorous stirring by a magnetic stirrer. Then, poly(1-ethenylpyrrolidin-2-one) $\left(M_{r}=12000\right)$ was added to the reaction mixture. This mixture was again under vigorous stirring for $30 \mathrm{~min}$. After, the autoclave was sealed and maintained at $190^{\circ} \mathrm{C}$ for $18 \mathrm{~h}$.
As soon as the reaction was completed, the autoclave was naturally cooled to room temperature. Finally, a dark gray precipitate was taken out by centrifuging and washed with deionized water and ethyl alcohol several times and then dried at $80^{\circ} \mathrm{C}$ for $8 \mathrm{~h}$.

Spark plasma sintering method by using a SPS25/10 system was applied to compact the synthesized powder at pressure of $40 \mathrm{MPa}$ and sintering time of $150 \mathrm{~s}$, resulting in the $\varnothing 20 \mathrm{~mm} \times 15 \mathrm{~mm}$ cylinders. Different SPS temperatures, $T_{S}$, equal to 690,720 , $735,750,780$, and $810 \mathrm{~K}$ were used. The $2 \times 2 \times$ $10 \mathrm{~mm}$ bars and the $\varnothing 10 \times 2 \mathrm{~mm}$ disks were cut along in-plane and out of plane directions of the cylinders.

Density of the samples sintered at different temperatures was measured by the Archimedes' method. To determine both crystal structure and phase composition of the starting powder and the bulk materials, $\mathrm{X}$-ray diffraction (XRD) analysis was performed by a Rigaku Ultima IV diffractometer with $\mathrm{Cu} K_{\alpha}$-radiation. To study grain structure features of the bulk samples, a scanning electron Nova NanoSEM 450 microscope (SEM) was applied. The specific electrical resistivity, $\rho$, and the Seebeck coefficient, $S$, of the bar samples were measured by using a ZEM-3 system. To determine the total thermal conductivity, $k_{t}$, of the disk-shaped samples, a TC-1200 system using a laser flash method was applied, too.

\section{EXPERIMENTAL RESULTS}

XRD pattern for the starting $\mathrm{Bi}_{1.9} \mathrm{Gd}_{0.1} \mathrm{Te}_{3}$ powder taken at room temperature is presented in Fig. 1a. The starting powder was found to be a single hexagonal phase characteristic for pure $\mathrm{Bi}_{2} \mathrm{Te}_{3}$ (PDF no. 01-0894302). Diffraction peaks can be correctly indexed with the standard diffraction planes corresponding to the space symmetry $R \overline{3} m$ group.

As was mentioned above, to prepare the bulk samples with various microstructures and thermoelectric properties, the starting powder was spark-plasma-sintered at $T_{S}=690,720,735,750,780$, and $810 \mathrm{~K}$ and for the same time (150 s) and under the same pressure (40 MPa). Density of all the samples sintered at different temperatures excepting $T_{S}=690$ was found to be weakly $T_{S}$-dependent and happened equal to $\sim 7.15 \mathrm{~g} \mathrm{~cm}^{-3}$, that is $\sim 93 \%$ of theoretical value of the $\mathrm{Bi}_{2} \mathrm{Te}_{3}$ density $\left(7.7 \mathrm{~g} / \mathrm{cm}^{3}\right)$. Density of the sample sintered at $T_{S}=690$ was equal to $6.62 \mathrm{~g} \mathrm{~cm}^{-3}$ (86\%). The density values for all the samples are collected in Table 1.

Like XRD pattern of the starting powder, all XRD patterns for the bulk samples sintered at different temperatures are also corresponding to the space symmetry $R \overline{3} m$ group. The lattice $a$ and $c$ parameters estimated by the Rietveld refinement are equal to 4.384 and $30.449 \AA$, respectively, for all the samples. That is, 
no $T_{S}$-effect on the crystal structure of the $\mathrm{Bi}_{1.9} \mathrm{Gd}_{0.1} \mathrm{Te}_{3}$ samples was found.

It was found that all the bulk samples are highly textured. This texturing is expressed as a big enough difference in the XRD patterns for the bulk samples collected on surfaces oriented either perpendicularly or parallel to the applied SPS pressuring. As an example, these XRD patterns taken for the bulk sample sintered at $T_{S}=750 \mathrm{~K}$ are shown in Figs. $1 \mathrm{~b}$ and $1 \mathrm{c}$, respectively. All the XRD peaks can be assigned to rhombohedral $R \overline{3} m$ structure, i.e. positions of the peaks are independent on the pressing direction. However, the intensities of some peaks were found to be strongly dependent on this direction. The intensities of the $(00 l)$ peaks are clearly enhanced in the XRD pattern taken for surface oriented perpendicular to the pressing direction (Fig. 1b), whereas in the XRD pattern taken for other pressing direction enhancing in the intensities is already occurred for the (11l) peaks (Fig. 1c). Such kind of intensity redistribution is usually attributed to forming a lamellar texture with preferential grain orientation along the pressing direction.

A degree of preferential grain orientation for the textured sample can be estimated in terms of the orientation factor, $F$, given by $[18,19]$

$$
F=\frac{p-p_{0}}{1-p_{0}}
$$

with $p$ and $p_{0}$ expressed as

$$
p=\frac{I(00 l)}{\Sigma I(h k l)} \quad \text { and } \quad p_{0}=\frac{I_{0}(00 l)}{\Sigma I_{0}(h k l)},
$$

where the $I$ and $I_{0}$ intensities are corresponding to oriented (textured) and non-oriented (non-textured) samples, respectively. Ideally, $F=1$ is for completely oriented sample similar to single crystal, whereas $F=0$ is for completely non-oriented sample similar to a powder or a grained material with completely random grain orientation.

To estimate the $F$ factor of the samples sintered at different $T_{S}$, the XRD patterns in Figs. $1 \mathrm{~b}$ and $1 \mathrm{c}$ were used to calculate the $p$ and $p_{0}$ values. The $F$ values estimated are presented in Table 1, too. One can see that firstly $F$ is slightly increasing with increasing $T_{S}$ from 0.59 (at $690 \mathrm{~K}$ ) up to 0.68 (at $750 \mathrm{~K}$ ), but at further increasing in $T_{S}$, the orientation factor starts to be decreasing down to 0.58 (at $810 \mathrm{~K}$ ). Hence, all the bulk samples are highly textured with preferential grain orientation along $c$-axis (or along the 001 direction), and the orientation factor is weak $T_{S}$-dependent.

Texturing observed is related to crystal structure features of the $\mathrm{Bi}_{2} \mathrm{Te}_{3}$-based compounds. As was mentioned above, these compounds have space $R \overline{3} \mathrm{~m}$ symmetry and unit cell parameters measured along the rhombohedral $c$-axis (the $c$ parameter) or within the $a-b$ plane oriented perpendicular to the $c$-axis (the $a$ and $b$ parameters) are very different from each other.
Table 1. The density, orientation factor and average grain sizes for the directions oriented parallel and perpendicular SPS-pressing directions

\begin{tabular}{c|c|c|c|c}
\hline$T_{S}, \mathrm{~K}$ & $\rho, \mathrm{g} \mathrm{cm}^{-3}$ & $F$ & $D_{\|}, \mathrm{nm}$ & $D_{\perp}, \mathrm{nm}$ \\
\hline 690 & 6.62 & 0.59 & 100 & 580 \\
720 & 7.25 & 0.60 & 110 & 645 \\
750 & 7.17 & 0.68 & 145 & 865 \\
780 & 7.15 & 0.65 & 300 & 1115 \\
810 & 7.05 & 0.58 & 805 & 1775 \\
\hline
\end{tabular}

For instance, the room temperature parameters for $\mathrm{Bi}_{2} \mathrm{Te}_{3}$ are $c=30.487 \AA$ and $a=b=4.384 \AA$. Such a big difference in the $c$ and $a=b$ parameters is originated from crystal structure anisotropy due to mainly combination of various types of bonding between atoms. The $\mathrm{Bi}_{2} \mathrm{Te}_{3}$ crystal structure is known to be consisted of 15 layers stacked along the $c$-axis and presents the combination of three hexagonal layer stacks of the composition in which each set consists of five atoms $\left(\mathrm{Te}_{1}-\mathrm{Bi}-\mathrm{Te}_{2}-\mathrm{Bi}-\mathrm{Te}_{1}\right)$ [1]. The bonding within the $\mathrm{Te}_{1}-\mathrm{Bi}-\mathrm{Te}_{2}-\mathrm{Bi}-\mathrm{Te}_{1}$ layer is assumed to be covalent, whereas the $\mathrm{Te}_{1}-\mathrm{Te}_{1}$ layers are bonded by the van der Waals forces.

Figure 2 shows the SEM-images of grain structures recorded on fractured surfaces oriented perpendicularly to the pressing direction. Only the samples sintered at $T_{S}=690,750$, and $810 \mathrm{~K}$ are used in this figure. One can see that the grains form ordered lamellar structure and lamellar sheets lie in plane perpendicular to the pressing direction. So, the SEM images in Fig. 2 are consistent with the XRD results (Fig. 1).

The lamellar sheets in Fig. 2 consist of the grains elongated in plane perpendicular to the pressing direction. In this case, the grain sizes in the direction parallel and perpendicular to the pressing direction are rather different. To estimate the average grain size, $\bar{D}$, in both directions, the histograms of the grain size distribution for the samples sintered at different $T_{S}$ were plotted and analyzed in frames of a lognormal unimodal distribution [36]. The $\bar{D}$ values estimated for directions parallel $\left(D_{\|}\right)$and perpendicular $\left(D_{\perp}\right)$ to the pressing direction are given in Table 1 . The average grain size measured along the pressing direction (or along the $c$-axis) is much less as compared to the average grain size measured in other direction. Besides, both average sizes are steady increasing with increasing $T_{S}$.

Now let us consider the $T_{S}$-effect on the thermoelectric properties of the samples studied. First of all, it should be recalled that in the highly textured $\mathrm{Bi}_{2} \mathrm{Te}_{3}$-based compounds, these properties are anisotropic [15-24]. For the SPS-textured $\mathrm{Bi}_{1.9} \mathrm{Gd}_{0.1} \mathrm{Te}_{3}$ compounds, we could find a strong anisotropy in the specific electrical resistivity and the total thermal con- 


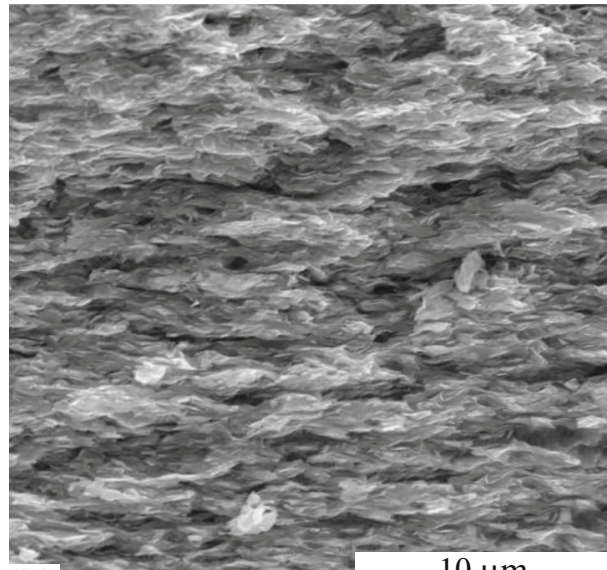

(a)

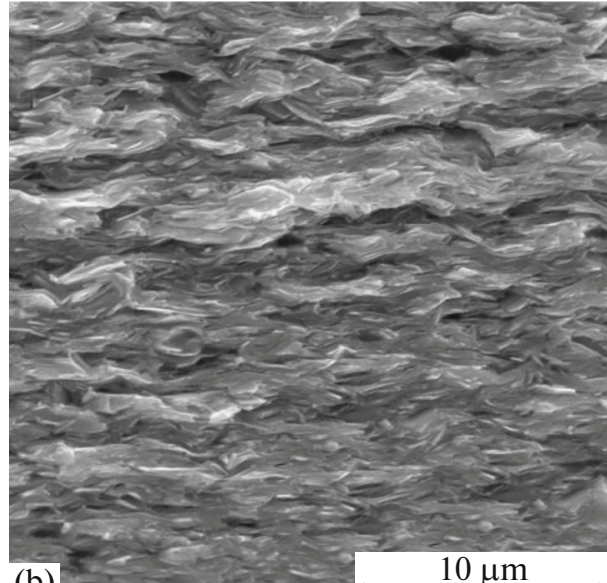

(b)

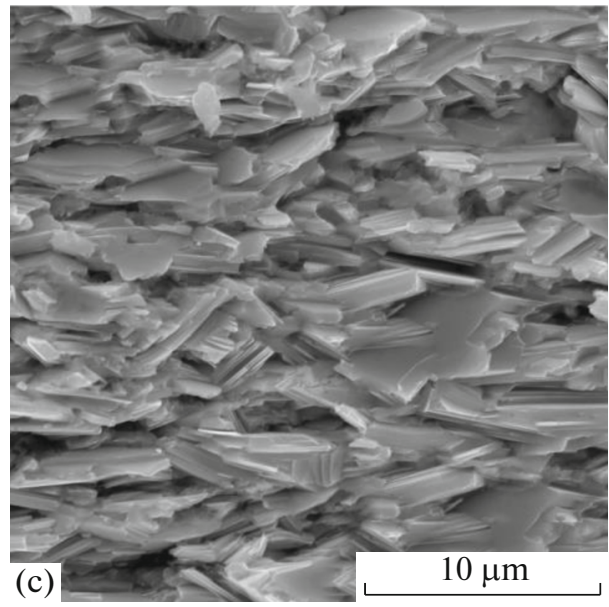

Fig. 2. The SEM images taken on the fractured surfaces perpendicular to the pressing direction for $T_{S}=690$ (a), 750 (b), and $810 \mathrm{~K}$ (c).

ductivity at measurements along directions parallel and perpendicular to the SPS-pressing direction. The thermoelectric properties for the perpendicular measuring orientation happen to be better as compared to those for the parallel measuring orientation. Therefore, in this paper analysis of the $T_{S}$-effect on the ther- moelectric properties of the $\mathrm{Bi}_{1.9} \mathrm{Gd}_{0.1} \mathrm{Te}_{3}$ compound will be limited by only data corresponding to the perpendicular measuring orientation, that in turn characterize the improving in the thermoelectric efficiency. Total comparative analysis of changing in the thermoelectric properties of the $\mathrm{Bi}_{1.9} \mathrm{Gd}_{0.1} \mathrm{Te}_{3}$ samples sintered at different temperatures for both measuring orientations will be presented elsewhere later.

Figure 3a shows the $\rho(T)$ dependences taken within the temperature $280-620 \mathrm{~K}$ range for the samples sintered at different $T_{S}$. As is seen, $\rho$ is gradually increasing with increasing temperature for all the samples. This behavior is characteristic of metals or degenerate semiconductors and usually can be attributed to a high-temperature carrier's scattering by phonons resulting in decreasing in carrier's mobility, when temperature increases.

The specific electrical resistivity was found to be $T_{S}$-dependent property of the samples. Within whole temperature range excepting high temperatures above $\sim 450 \mathrm{~K}$ for the samples sintered at 780 and $810 \mathrm{~K}, \rho$ is gradually decreasing with increasing $T_{S}$. This tendency is obviously correlated with gradual increasing in the average grain size that was also observed with increasing sintering temperature. In this case, an additional carrier's scattering by the grain boundaries resulting in decreasing in the carrier's mobility should be considered as mechanism responsible for the changing in $\rho$ in the samples sintered at different $T_{S}$.

The $T_{S}$-effect on $\rho$ above $\sim 450 \mathrm{~K}$ is rather complicated and its analysis is apart from the main aim of this paper. However, it should be noted that at these high temperatures, an intrinsic conductivity due to a thermal excitation of the carriers from valence band to conduction band usually starts to be developing in the $\mathrm{Bi}_{2} \mathrm{Te}_{3}$-based compounds.

The temperature dependences of the Seebeck coefficient for all the samples are shown in Fig. 3b. The Seebeck coefficient naturally has a negative sign characteristic of $n$-type conductivity in the samples studied. In contrast to $T_{S}$-effect on $\rho, S$ is nonmonotonically changing with increasing $T_{S}$. With increasing $T_{S}$ from 690 up to $750 \mathrm{~K}, S$ is gradually increasing, but above $T_{S}=750 \mathrm{~K}$ the Seebeck coefficient starts to be decreasing. So, the highest $S$ value is observed for the sample sintered at $750 \mathrm{~K}$. It should be also noted that all the $S(T)$ curves have the maxima centered at temperature of $\sim 450 \mathrm{~K}$. Usually, these maxima are originated from a bipolar effect and are typical for the doped $\mathrm{Bi}_{2} \mathrm{Te}_{3}$ and $\mathrm{Bi}_{2} \mathrm{Te}_{3}$-based compounds [31-35]. The bipolar effect is observed, when electrons and holes are simultaneously present in semiconductor. The thermal excitation of the carrier's due to the intrinsic conductivity does not change the majority carrier's concentration too much, whereas the 
minority carrier's concentration will be remarkably increasing. The $S$ sign for the electron conductivity is known to be negative, but the hole conductivity is already characterized by the positive $S$ sign. Therefore, a competition of two $S$ contributions having the opposite signs will result in the $S(T)$ maxima in the $S(T)$ curves.

The temperature dependences of the power factor for all the samples calculated as $S^{2} / \rho$ are shown in Fig. 3c. These dependences account for the $\rho$ and $S$ contributions. Owing to the highest Seebeck coefficient and the low enough specific electrical resistivity, the highest power factor is observed for the sample sintered at $750 \mathrm{~K}$. And vice versa, the sample sintered at $690 \mathrm{~K}$ has the highest $\rho$ and one of the lowest $S$ among all the samples, that in turn results in the lowest power factor in this sample.

The temperature dependences of the total thermal conductivity, $k_{t}$, of the samples sintered at different $T_{S}$ are presented in Fig. 4a. All the $k_{t}(T)$ curves are rather complicated. The minima located at $\sim 430 \mathrm{~K}$ can be attributed to changing in the thermal conductivity mechanisms. Generally, contributions from crystal lattice, carriers and bipolar conductivity should be taken into account to analyze in detail the changing in the $k_{t}(T)$ curves for the samples sintered at different $T_{S}$. For the $\mathrm{Bi}_{2} \mathrm{Te}_{3}$-based compounds, the crystal lattice contribution is main one at low temperatures below the $k_{t}(T)$ minimum temperature, whereas the bipolar conductivity contribution results in $k_{t}$ growth above the minimum temperature [33-35]. Detailed analysis of the $T_{S}$-effect on all the thermal conductivity contributions of the $\mathrm{Bi}_{1.9} \mathrm{Gd}_{0.1} \mathrm{Te}_{3}$ samples sintered at different temperatures is in progress. It is more interesting that similar to the specific electrical resistivity, $k_{t}$ is also strongly $T_{S}$-dependent. But in contrast to the $\rho-T_{S}$ behavior, $k_{t}$ is gradually increasing with increasing $T_{S}$. It should be mentioned especially that this $k_{t}-T_{S}$ tendency is also correlated with gradual increasing in the average grain size. Since the $k_{t}-T_{S}$ tendency is qualitatively agreed with the changes in the average grain size, it means that the grain boundaries can be believed to be effective scattering centers not only for the electrons (Fig. 3a), but also for the phonons.

Finally, the $\rho, S$, and $k$ values were used to plot the $Z T(T)$ dependences for the samples sintered at different $T_{S}$ (Fig. 5).

All the $Z T(T)$ dependences have clear maxima positioned at temperature of $\sim 430 \mathrm{~K}$. These maxima can be obviously related to onset of the intrinsic conductivity at high temperatures. The intrinsic conductivity is harmful for the thermoelectric efficiency enhancement. The $T_{S}$-effect on the thermoelectric

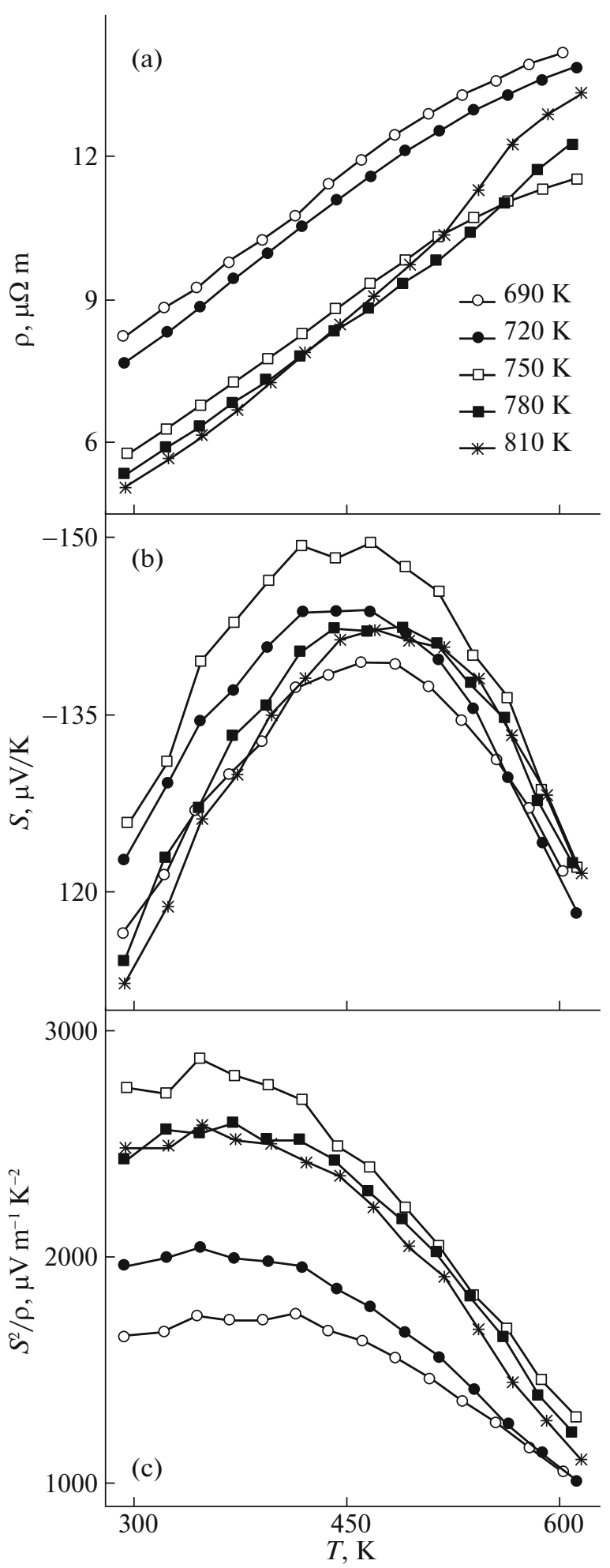

Fig. 3. The temperature dependences of $\rho$ (a), $S$ (b) and $S^{2} / \rho$ (c) for the different $T_{S}$.

figure-of-merit of the grained $\mathrm{Bi}_{1.9} \mathrm{Gd}_{0.1} \mathrm{Te}_{3}$ compounds is clearly expressed. The highest $Z T$ value equal to $\sim 0.73$ was observed for the sample SPS-sin- 


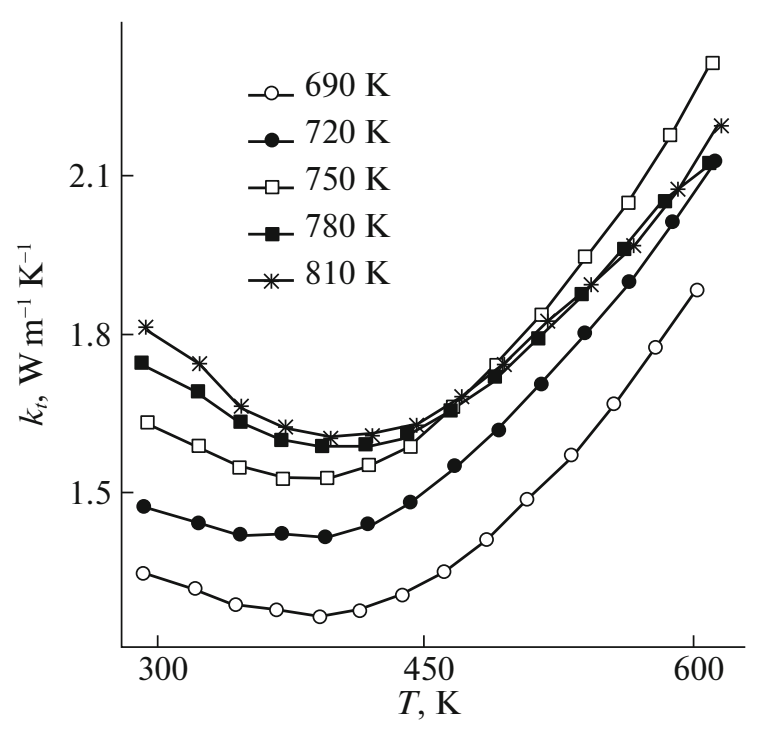

Fig. 4. The temperature dependences of $k_{t}$ for the different $T_{S}$.

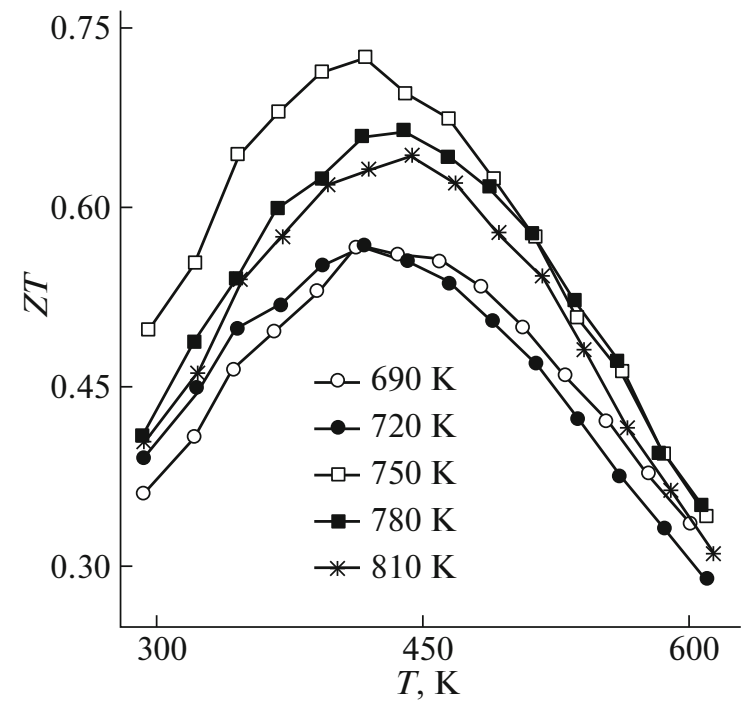

Fig. 5. The temperature dependences of $Z T$ for the different $T_{S}$.

tered at $T_{S}=750 \mathrm{~K}$. Although this sample possesses mean thermal conductivity value among other samples, its highest power factor is dominant source favoring to the $Z T$ maximization.

\section{CONCLUSIONS}

Thus, the spark plasma sintering temperature can be considered as one of technological variables tuning the thermoelectric properties of the $n$-type grained $\mathrm{Bi}_{1.9} \mathrm{Gd}_{0.1} \mathrm{Te}_{3}$ thermoelectric.

\section{ACKNOWLEDGMENTS}

All of studies were carried out by the scientific equipment of joint research center "Technologies and Materials" at the Belgorod State University.

\section{FUNDING}

This research was funded by the Ministry of Education and Science, Russia, under grant no. 11.3719.2017/PCh (11.3719.2017/4.6).

\section{REFERENCES}

1. G. S. Nolas, J. Sharp, and H. J. Goldsmid, Thermoelectrics Basic Principles and New Materials Developments (Springer, Berlin, 2001).

2. H. J. Goldsmid, Materials 7, 2577 (2014).

3. H. Kitagawa, T. Nagamori, T. Tatsuta, T. Kitamura, Y. Shinohara, and Y. Noda, Scr. Mater. 49, 309 (2003).

4. D. B. Hyun, T. S. Oh, J. S. Hwang, J. D. Shim, and N. V. Kolomoets, Scr. Mater. 40, 49 (1998).

5. S. Miura, Y. Satob, K. Fukuda, K. Nishimura, and K. Ikeda, Sci. Eng. A 277, 244 (2000).

6. O. Ivanov, O. Maradudina, and R. Lyubushkin, J. Alloys Compd. 586, 679 (2014).

7. W. Liu, X. Yan, G. Chen, and Z. Ren, Nano Energy 1, 42 (2012).

8. Y. Li, J. Jiang, G. Xu, W. Li, L. Zhou, Y. Li, and P. Cui, J. Alloys Compd. 480, 954 (2009).

9. S. S. Kim, S. Yamamoto, and T. Aizawa, J. Alloys Compd. 375, 107 (2004).

10. Y. Morisaki, H. Araki, H. Kitagawa, M. Orihashi, K. Hasezaki, and K. Kimura, Mater. Trans. 46, 2518 (2005).

11. X. K. Duan, K. G. Hu, D. H. Ma, W. N. Zhang, Y. Z. Jiang, and S. C. Guo, Rare Met. 34, 770 (2015).

12. P. Srivastava and K. Singh, Mater. Lett. 136, 337 (2014).

13. B. Jarivala, D. Shah, and N. M. Ravindra, J. Electron. Mater. 44, 1509 (2015).

14. O. Ivanov, O. Maradudina, and R. Lyubushkin, Mater. Char. 99, 175 (2015).

15. O. Ben-Yehuda, R. Shuker, Y. Gelbstein, Z. Dashevsky, and M. P. Dariel, J. Appl. Phys. 101, 113707 (2007).

16. J. J. Shen, L. P. Hu, T. J. Zhu, and X. B. Zhao, Appl. Phys. Lett. 99, 124102 (2011).

17. X. Yan, B. Poudel, W. S. Liu, G. Joshi, H. Wang, Y. Lan, D. Wang, G. Chen, and Z. F. Ren, Nano Lett. 10, 3373 (2010).

18. S. D. Bhame, D. Pravarthana, W. Prellier, and J. G. Noudem, Appl. Phys. Lett. 102, 2190 (2013).

19. X. A. Fan, J. Y. Yang, R. G. Chen, H. S. Yun, W. Zhu, S. Q. Bao, and X. K. Duan, J. Phys. D: Appl. Phys. 39, 740 (2006).

20. J. Jiang, L. Chen, S. Bai, Q. Yao, and Q. Wang, Mater. Sci. Eng. B 117, 334 (2005). 
21. Q. Lognon, F. Gascoin, O. I. Lebedev, L. Lutterotti, S. Gascoin, and D. Chateigner, J. Am. Ceram. Soc. 97, 2038 (2014).

22. J.-J. Shen, T.-J. Zhu, X.-B. Zhao, S.-N. Zhang, S.-H. Yang, and Z.-Z. Yin, Energy Environ. Sci. 3, 1519 (2010).

23. C. Andre, D. Vasilevskiy, S. Turenne, and R. A. Masut, J. Phys. D.: Appl. Phys. 44, 235401 (2011).

24. A. Vasil'ev, M. Yaprintsev, O. Ivanov, and E. Danshina, Solid State Sci. 84, 28 (2018).

25. J. Yang, F. Wu, Z. Zhu, L. Yao, H. Song, and X. Hu, J. Alloys Compd. 619, 401 (2015).

26. X. H. Ji, X. B. Zhao, Y. H. Zhang, B. H. Lu, and H. L. Ni, J. Alloys Compd. 387, 282 (2005).

27. F. Wu, H. Song, J. Jia, and X. Hu, Prog. Natl. Sci. Mater. Int. 23, 408 (2013).

28. F. Wu, W. Shi, and X. Hu, Electron. Mater. Lett. 11, 127 (2015).
29. X. H. Ji, X. B. Zhao, Y. H. Zhang, B. H. Lu, and H. L. Ni, Mater. Lett. 59, 682 (2005).

30. F. Wu, H. Z. Song, J. F. Jia, F. Gao, Y. J. Zhang, and X. Hu, Phys. Status Solidi A 210, 1183 (2013).

31. W. Y. Shi, F. Wu, K. L. Wang, J. J. Yang, H. Z. Song, and X. J. Hu, Electron. Mater. 43, 3162 (2014).

32. X. B. Zhao, Y. H. Zhang, and X. H. Ji, Inorg. Chem. Commun. 7, 386 (2004).

33. O. Ivanov, M. Yaprintsev, R. Lyubushkin, and O. Soklakova, Scr. Mater. 146, 91 (2018).

34. M. Yaprintsev, A. Vasil'ev, and O. Ivanov, J. Eur. Ceram. Soc. 39, 1193 (2019).

35. O. Ivanov and M. Yaprintsev, Mater. Res. Express 5, 015905 (2018).

36. F. J. Humphreys and M. Hatherly, Recrystallization and Related Annealing Phenomena (Elsevier, Oxford, 2004). 